Original paper

\title{
Impact of interfractional changes in head and neck cancer patients on the delivered dose in intensity modulated radiotherapy with protons and photons
}

\author{
Birgit Sabine Müller a, b, *, Marciana Nona Duma ${ }^{\text {a }}$, Severin Kampfer ${ }^{\text {a, b }}$, Simeon Nill ${ }^{\text {c }}$, \\ Uwe Oelfke $^{\mathrm{c}}$, Hans Geinitz ${ }^{\mathrm{d}, \mathrm{e}}$, Jan Jakob Wilkens ${ }^{\text {a, b }}$ \\ a Department of Radiation Oncology, Technische Universität München, Klinikum rechts der Isar, Munich, Germany \\ b Physik-Department, Technische Universität München, Munich, Germany \\ c Joint Department of Physics at The Institute of Cancer Research and The Royal Marsden NHS Foundation Trust, London, UK \\ ${ }^{d}$ Department of Radiation Oncology, Krankenhaus der Barmherzigen Schwestern Linz, Linz, Austria \\ e Medical Faculty, Johannes Kepler University, Linz, Austria
}

\section{A R T I C L E I N F O}

\section{Article history:}

Received 21 November 2014

Received in revised form

3 February 2015

Accepted 7 February 2015

Available online 25 February 2015

\section{Keywords:}

IGRT

IMPT

IMXT

Adaptive planning

\begin{abstract}
A B S T R A C T
Purpose: To investigate the influence of interfractional changes on the delivered dose of intensity modulated proton (IMPT) and photon plans (IMXT).

Methods and materials: Five postoperative head and neck cancer patients, previously treated with tomotherapy at our institute, were analyzed. The planning study is based on megavoltage (MV) control images. For each patient one IMPT plan and one IMXT plan were generated on the first MV-CT and recalculated on weekly control MV-CTs in the actual treatment position. Dose criteria for evaluation were coverage and conformity of the planning target volume (PTV), as well as mean dose to parotids and maximum dose to spinal cord.

Results: Considerable dosimetric changes were observed for IMPT and IMXT plans. Proton plans showed a more pronounced increase of maximum dose and decrease of minimum dose with local underdosage occurring even in the center of the PTV (worst IMPT vs. IMXT coverage: $66.7 \%$ vs. $85.0 \%$ ). The doses to organs at risk (OARs) increased during the treatment period. However, the OAR doses of IMPT stayed below corresponding IMXT values at any time. For both modalities treatment plans did not necessarily worsen monotonically throughout the treatment.

Conclusions: Although absolute differences between planned and reconstructed doses were larger in IMPT plans, doses to OARs were higher in IMXT plans. Tumor coverage was more stable in IMXT plans; IMPT dose distributions indicated a high risk for local underdosage during the treatment course.

() 2015 Associazione Italiana di Fisica Medica. Published by Elsevier Ltd. This is an open access article under the CC BY-NC-ND license (http://creativecommons.org/licenses/by-nc-nd/4.0/).
\end{abstract}

\section{Introduction}

Interfractional changes of patient anatomy, due to patient weight loss and shrinkage of the tumor and structures nearby, as well as variations in daily set-up, are of concern in radiotherapy. In order to retain dosimetric quality during the treatment phase,

* Corresponding author. Department of Radiation Oncology, Technische Universität München, Klinikum rechts der Isar, Ismaninger Straße 22, 81675 München, Germany. Tel.: +49 (0) 894140 6231; fax: +49 (0) 8941404881.

E-mail address: Birgit.Mueller@lrz.tu-muenchen.de (B.S. Müller). image guided radiotherapy (IGRT) and adaptive planning are a part of clinical practice.

Head and neck patients are known to be subject to severe anatomical changes throughout the treatment course and therefore benefit from frequent controls [1-6]. Their treatment plans are often strongly modulated, i.e. featuring steep dose gradients within fields which may even be more sensitive to geometrical changes $[3,5,7]$. So far there is no standard solution on how often controls have to be performed, when to adapt a plan and which patients benefit from plan adaptions.

Depending on the applied irradiation technique anatomical changes may have different degrees of impact. Dose distributions in intensity modulated proton therapy (IMPT) are often superior 
compared to intensity modulated photon plans (IMXT) [3,8-13]. The Bragg peak and the well-defined range of the protons allow better sparing of healthy tissue and higher conformity of the target volume. Nevertheless these advantageous physical properties of protons can also be a risk in clinical practice [14-20].

Due to the different physical characteristics of photons and protons, e.g. the sensitivity of depth dose curves to density changes of traversed matter, it is of interest how sensitive IMPT and IMXT plan qualities are to interfractional anatomical changes of patients and how relevant IGRT is for both techniques.

This study focuses on IMPT and IMXT treatment plans of head and neck cancer patients that were not adapted "in time". We present an analysis of dosimetric alterations due to interfractional changes by recalculating the initial plans on control images at different times during the treatment period.

\section{Material and methods}

\section{Patient characteristics}

This retrospective planning study was performed on data of five postoperative head and neck cancer patients ( 1 hypopharynx, 2 tonsil, 1 oral cavity, 1 larynx) treated by tomotherapy (Accuray Inc., Madison, WI) at our institute. Four of them underwent radiochemotherapy. The clinical target volume (CTV) was defined as the GTV (gross tumor volume) on the preoperative imaging, including the postoperative region, plus a margin of $10 \mathrm{~mm}$. The elective nodal-CTV was defined according to Grégoire et al. and Chao et al. [21,22]. The initial planning target volumes $\left(\mathrm{PTV}=\mathrm{CTV}+5 \mathrm{~mm}\right.$ safety margin) ranged from $721.7 \mathrm{~cm}^{3}$ to $1296.7 \mathrm{~cm}^{3}$.

Patients were immobilized with a head and shoulder mask. Daily control megavoltage (MV) CTs were acquired and merged with the planning kilovoltage $\mathrm{CT}$ scan $(\mathrm{kV}-\mathrm{CT})$ before treatment. After registration by the bone and tissue registration algorithm provided by the tomotherapy unit, the automatic registration was manually corrected by staff members with regard to tumor coverage and spinal cord sparing. These images representing the actual treatment position served as basis for our planning study (Fig. 1).

For the contouring process on the MV-CTs the images were imported into the Eclipse planning system (Varian Medical Systems Inc., Palo Alto, CA). In order to minimize bias, the contouring process on the MV-CTs was done by the same physician for all CTs. The structures of the original PTV and organs at risk (OARs) of the $\mathrm{kV}-\mathrm{CT}$ were copied to the merged images and manually adapted to the new anatomy.

During the treatment period (approx. 6 weeks) all patients lost weight. The absolute amount varied during the treatment (e.g. by saline volume expansion during chemotherapy administration), and between patients from $2.0 \mathrm{~kg}$ to $12.8 \mathrm{~kg}$. Similar fluctuations

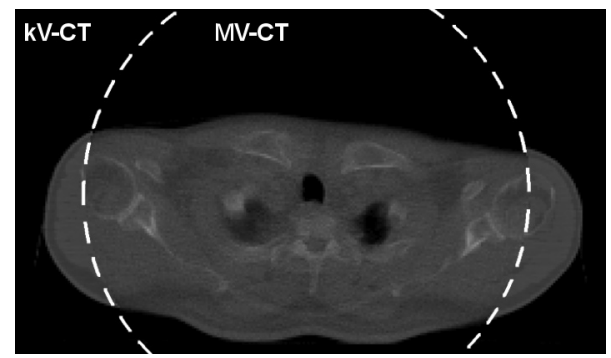

Figure 1. One slice of a merged CT consisting of a MV-image in the center and of small initial $\mathrm{kV}-\mathrm{CT}$ parts to fill up the area outside of the (smaller) field of view of the MV-CT. were observed for losses of PTV volumes. The mean PTV loss was $76.2 \pm 50.3 \mathrm{~cm}^{3}$ (corresponding to $7.0 \pm 4.6 \%$ of the initial volume) and the greatest loss $143.1 \mathrm{~cm}^{3}(12.9 \%)$.

\section{Retrospective planning}

Two plans were calculated for each patient, one IMPT and one IMXT plan. The retrospective planning for both modalities was carried out in a research version of the KonRad planning system, developed at German Cancer Research Center in Heidelberg [23].

Planning on MV-CTs required the adaption of look-up tables (LUT) to convert Hounsfield units into water equivalent depth/ relative stopping power. The LUT data published by Newhauser [24] served as basis for proton planning, for photon planning the clinically used tomotherapy data was implemented. To avoid any Hounsfield unit calibration issues due to $\mathrm{kV}$ - and MV-planning and associated LUTs all dose calculations were performed on MV-CTs, i.e. the first MV-CT was used as planning CT.

Proton plans were calculated using data of a virtual scanning machine with a constant lateral beam width of $2.5 \mathrm{~mm}$ standard deviation (Gaussian beam profile) at the patient surface. We selected three fields such that no proton beam traversed any parts of the $\mathrm{kV}-\mathrm{CT}$ : one posterior $\left(180^{\circ}\right)$ and two lateral oblique fields $\left(45^{\circ}, 315^{\circ}\right)$, with spot spacing of $\Delta x=\Delta y=\Delta z=0.3 \mathrm{~cm}$ within the PTV (3D spot scanning technique [25]). The fields were optimized simultaneously assigning the spot weights such that the combined dose distribution fulfilled the defined objectives as best as possible.

The IMXT plans (seven equidistant fields, $6 \mathrm{MV}$ ) were generated by simultaneous fluence optimization and direct translation into multileaf collimator (MLC) segments in step and shoot mode (leaf size: $0.5 \mathrm{~mm}$ ). Complete blocking of $\mathrm{kV}$-parts of the image was not possible. Since the geometry of the $\mathrm{kV}$ image was identical for each merged image and the influence of different LUTs for $\mathrm{kV}$ and MV on the photon dose is comparatively small, the relative error between CTs caused for IMXT planning can be neglected.

The planning and evaluation process were done on identical target structures for IMPT and IMXT. Typically, range uncertainties specific of IMPT can be addressed during the planning process by additional PTV margins adjusted to beam directions. It is therefore difficult to define appropriate target structures to compare IMPT and IMXT, as this depends on the individual study intent. Since our goal was to study the differences in dosimetric changes over the course of a treatment for each technique in any comparable target structure, the photon PTV (as clinically used in our department) served as the target volume here. The PTV accounts for setup errors, which - even with image guidance - can never be eliminated completely, and for internal organ motion which motivated us to evaluate the dose delivered to this volume (rather than e.g. to the CTV).

The initial plans were optimized by the same constraints (with individually adjusted penalty factors): first priority was given to PTV coverage, (prescribed dose: $50 \mathrm{~Gy}$ for photons, $50 \mathrm{~Gy}(\mathrm{RBE})$ for protons in 25 fractions). In the following all dose values are given in Gy referring to absolute absorbed dose in case of photons and relative biological effect ( $\mathrm{RBE}=1.1$ ) weighted dose in case of protons. Second priority was given to the OARs, with the focus on the spinal cord (maximum dose $D_{\max }<45 \mathrm{~Gy}$ ) and parotid glands (mean dose $\mathrm{D}_{\text {mean }}<26 \mathrm{~Gy}$ ). Sometimes objectives were not achieved and approached at the cost of the PTV. The obtained plans, one IMPT and one IMXT plan for each patient, were recalculated on five MV-CTs (5th, 10th, 15th, 20th, 25th fraction) that were registered to the actual treatment position as described above.

The resulting dose distributions were analyzed in terms of dose volume histograms (DVHs) and various indicators for the PTV and 
for OARs. Target coverage and conformity were analyzed by the following indices [26]:

Coverage Index : CovI $=V 95(P T V) / V(P T V)$

Conformity index : ConI $=1+$ V95(Normal Tissue $) / V 95(P T V)$

(V95: absolute volume in $\mathrm{cm}^{3}$ receiving $95 \%$ of the prescribed dose; normal tissue: patient contour excluding PTV)

As to the OARs, within this paper we will focus on the spinal cord and parotid glands. Further organs were considered, not obtaining further conclusions though. For the spinal cord we assessed the maximum, for the latter the mean dose. Maximum and minimum doses were evaluated as the maximum and minimum received by at least $1 \mathrm{~cm}^{3}$ of the structure.

\section{Results}

\section{Example patient}

While the PTV coverage was comparably satisfying, the dose distributions and DVHs indicated better target conformity and preservation of OARs in the initial IMPT plan as compared to the initial IMXT plan (Fig. 2, first subplots of Figs. 3-6).

Throughout the treatment the homogeneity and coverage of the PTV decreased and the areas of under- and overdosage increased for both plans. The resulted heterogeneity was far greater for proton than for photon plans (Figs. 2 and 7). While IMPT plans revealed underdosed spots also in the central PTV (e.g. compare recalculations on 25th CT, Fig. 2), cold spots in IMXT plans were located at the outer PTV regions.

For both techniques the location of hot spots was not necessarily restricted to the PTV but were also found outside - e.g. within the parotid gland. The steepness of the DVH of the PTV degraded for both plan types. Compared to the IMPT plans, the IMXT plans changed moderately. The quality of the DVHs did not worsen monotonically but the plots of the 15th CT, were superior to the 10th fraction for both types of plans.

\section{PTV of all patients}

The initial $D_{\max }\left(1 \mathrm{~cm}^{3}\right), D_{\min }\left(1 \mathrm{~cm}^{3}\right)$ and $D_{\text {mean }}$ of the PTV were similar for the five patients for IMPT and IMXT, except for $D_{\min }\left(1 \mathrm{~cm}^{3}\right)$ being slightly lower for photons (Fig. 3). The changes of doses during treatment were different though. The $D_{\text {mean }}$ was almost constant at $2 \mathrm{~Gy}$ for both plans. The $\mathrm{D}_{\max }\left(1 \mathrm{~cm}^{3}\right)$ increased and $D_{\min }\left(1 \mathrm{~cm}^{3}\right)$ decreased considerably for the IMPT plans, whereas this divergence was moderate for IMXT. Generally recalculated IMPT dose distributions showed extremer inhomogeneties (Figs. 2 and 7). In the IMPT plans spots of underdosage were located also in the center of the PTV while in IMXT they restrained to the boundary area. The suggested decline in homogeneity and coverage was further supported by the increasing trend of ConI and decreasing trend of CovI (Fig. 4). An exception were the results of patient 5 in fraction 15: the $D_{\min }\left(1 \mathrm{~cm}^{3}\right)$ was smaller in the photon than in the proton plan. Regarding the corresponding CovIs nonetheless this was superior to the IMPT plan.

Especially the assessed quantities of IMPT plans showed large standard deviations, i.e. great variations between patients (Figs. 3 , 4). The general trends (e.g. decreasing coverage) were still seen in the individual patient graphs.

\section{Organs at risk of all patients}

For both modalities an increasing trend of $D_{\max }\left(1 \mathrm{~cm}^{3}\right)$ to the spinal cord was observed, the absolute changes being greater for protons (Fig. 5). With $D_{\max }\left(1 \mathrm{~cm}^{3}\right)$ of the IMXT plans initiating at far higher values, no proton plan reached maxima in the range of the IMXT plans over the whole course.
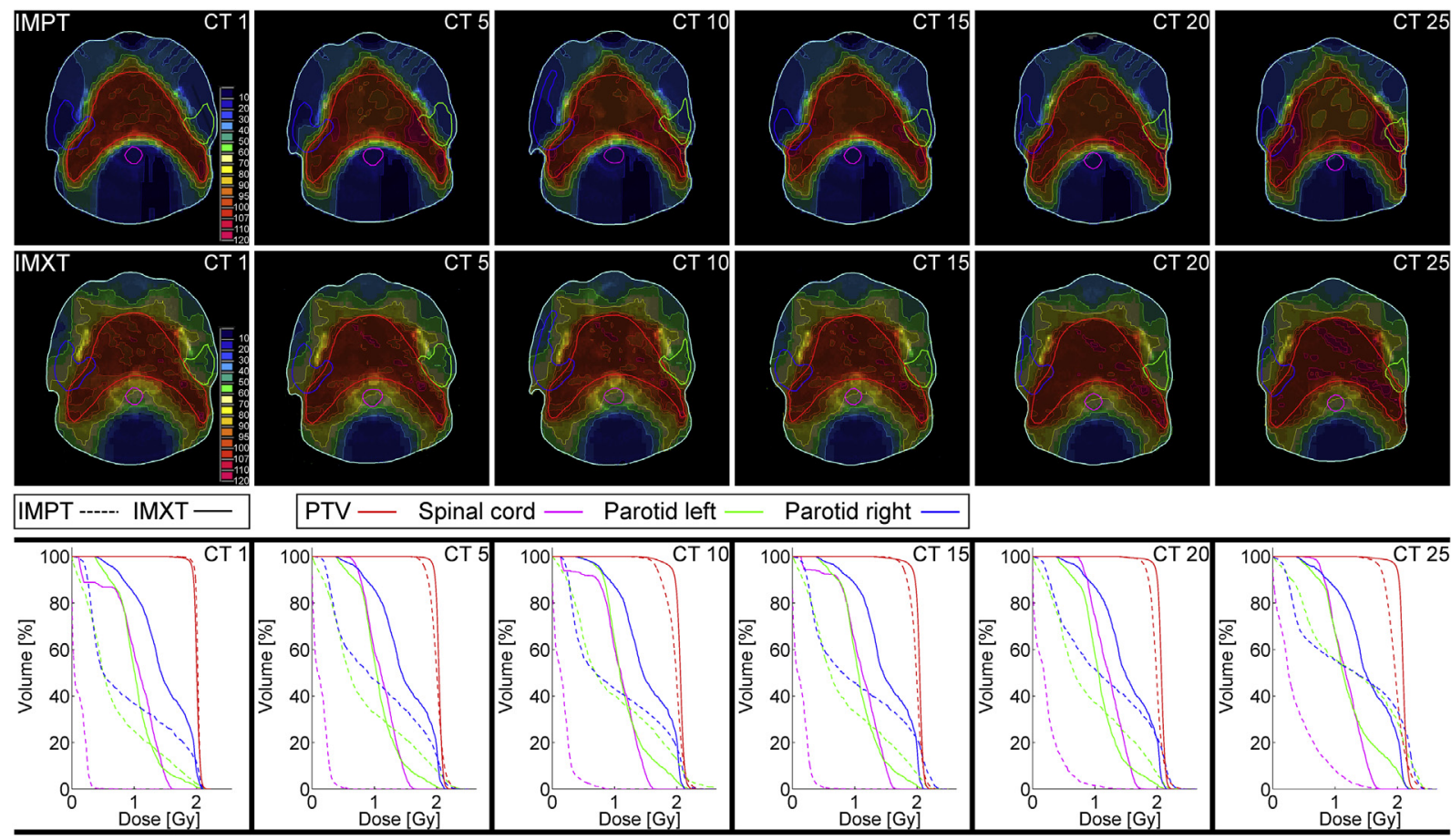

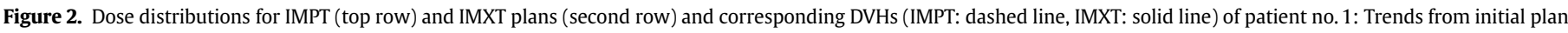
(CT1, left) to recalculations of the last fraction (right). (Normalization: $100 \%=2 \mathrm{~Gy}$; PTV: planning target volume). 


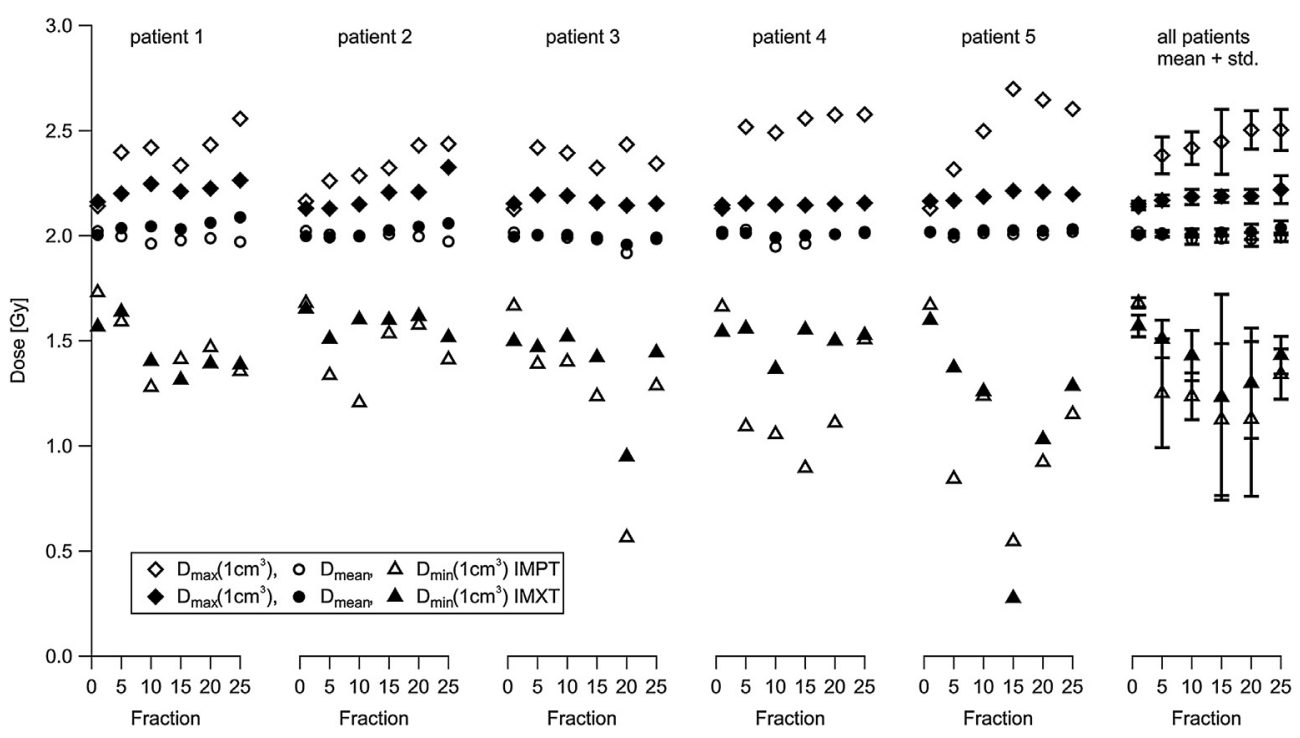

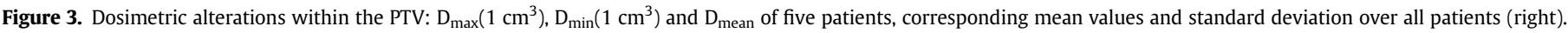

Parotid sparing was initially better in the IMPT plans, i.e. with IMXT less parotid tissue was spared (Fig. 6). Since the PTV was almost symmetrical there was no distinction between the ipsi- and contralateral parotid gland. Overall, both modalities had an increase in dose during treatment course, with smaller differences between the planned and recalculated doses for IMXT as compared to IMPT.

Some cases showed a decreasing $\mathrm{D}_{\text {mean }}$ (e.g. left parotid of 3rd patient) for IMPT and IMXT, likely due to different positioning. These findings suggest that optimizing parotid doses as low as possible is reasonable: even if defined dose limits cannot be obtained, the sum of actual delivered doses might be below the first calculated doses.

\section{Discussion}

Recently a related study was published by Kraan et al. [15] analyzing the impact of clinical uncertainties to IMPT plans of oropharynx patients. A variety of scenarios of potential errors on the basis of two clinical CTs per patient (planning and one control $\mathrm{CT}$ ) were simulated and the benefit of plan adaption was examined. Setup errors were simulated applying isocenter shifts and anatomical uncertainties were evaluated by recalculations on control CTs. The authors concluded that the combination of different errors can cause serious effects and recommended individual surveillance of anatomy, dose recalculations and plan adaptions. This recommendation is sustained by our study analyzing several control CTs during the treatment course displaying the consequences of anatomical changes as well as patient positioning uncertainties on the dose distribution during fractionated radiotherapy. Observed dose changes varied between individual patients which is also consistent to our findings.

Simone II et al. [3] compared adaptive to non-adaptive planning with IMPT and IMXT for head and neck patients. One result was that adaptive IMPT offers the most favorable dose quality. Our results agree with that, assuming that the patient received the calculated dose.

In both above cited studies it was assumed that the anatomy remained identical up to the day of the control CT and then until

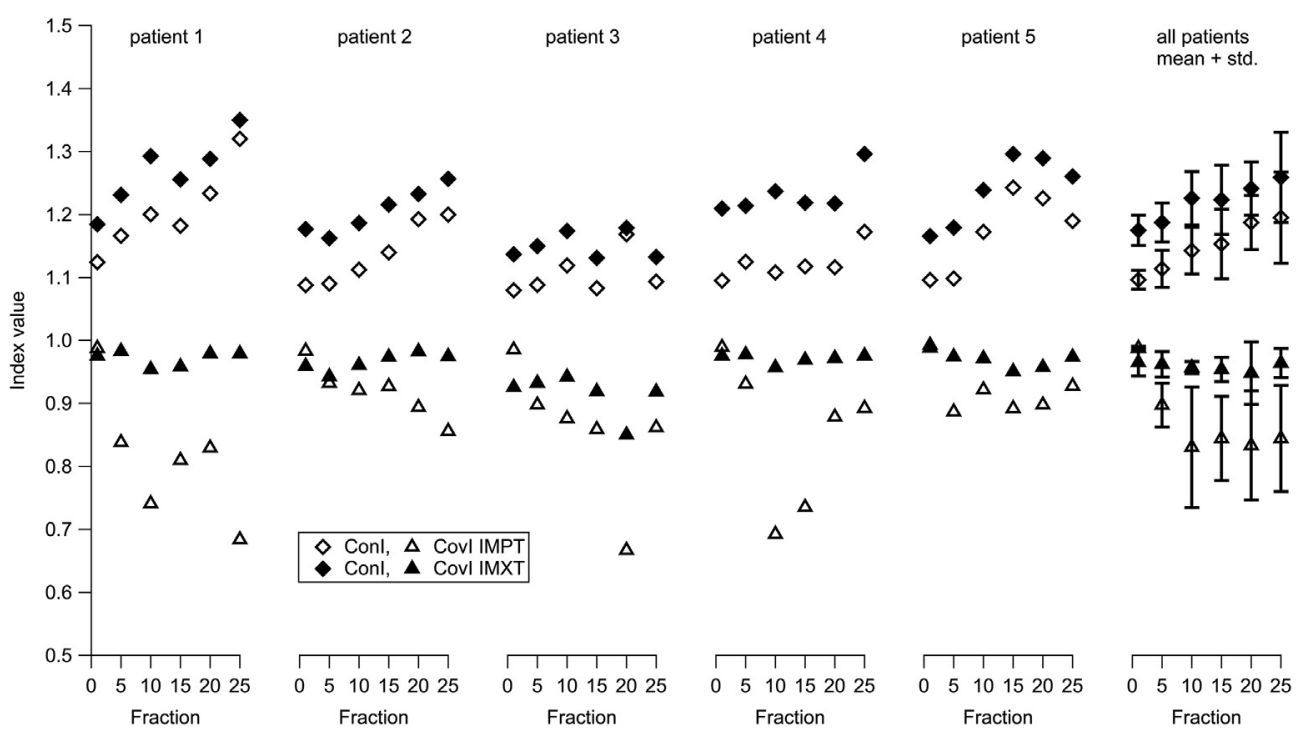

Figure 4. Trends of coverage (CovI) and conformity indices (ConI) for five patients, corresponding mean values and standard deviations (right). 


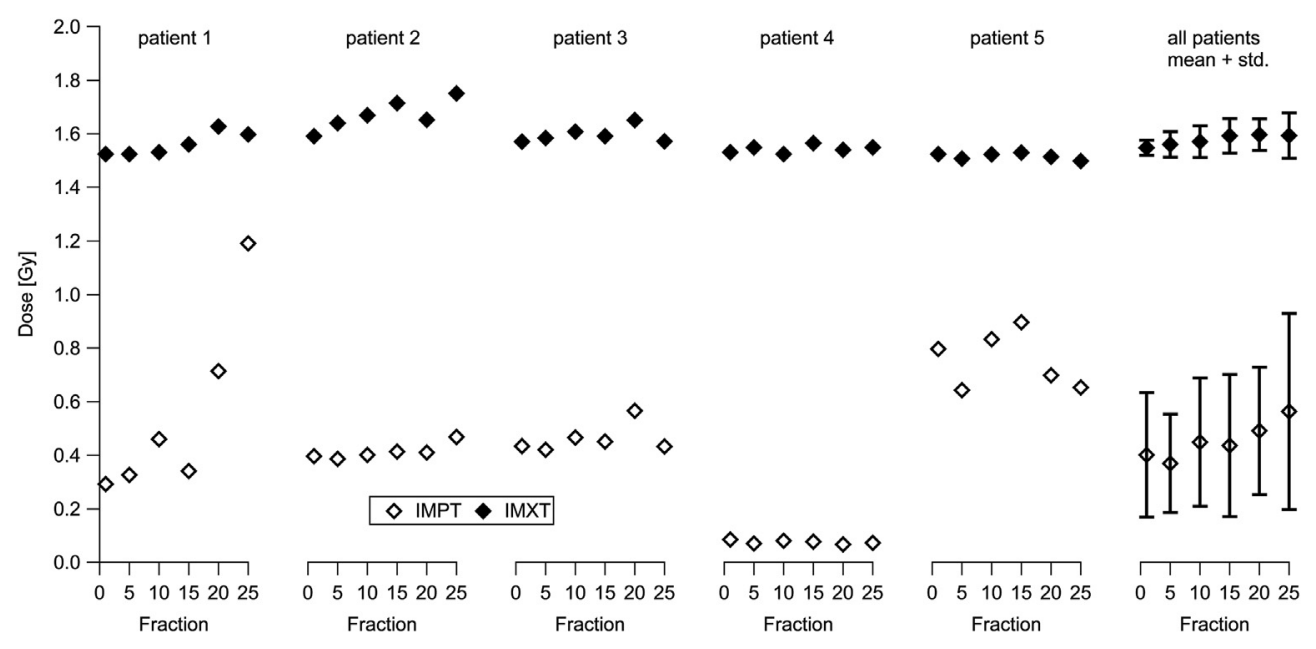

Figure 5. Trends of $D_{\max }\left(1 \mathrm{~cm}^{3}\right)$ to the spinal cord for five patients and corresponding mean values and standard deviations (right).

treatment finalization. Since patients undergo continuous anatomical changes there is a certain risk that the dose was not delivered as planned until the fraction where adaptation started. Our results obtained by recalculations on the 5th and 10th control $\mathrm{CT}$, indicate that adaption might already be relevant earlier during the treatment course. Our investigations complement these studies with patient data that reflects the actual clinical situation in positioning and anatomy in frequent intervals.

Contrary to the possible assumption that the anatomical changes and its dosimetric consequences worsen throughout the treatment, we showed that the decrease in quality is not necessarily monotonic. Some assessed values "improved" again at the following control CT, acquired five fractions later. Nevertheless the general trend was obviously indicating to worsen towards the end of the treatment, i.e. dosimetric quality indicators of treatment at the last fraction were worse than in the beginning. The search for explanations of the non-existing monotony is challenging as well as the search of correlations between dose criteria and weight loss which could serve as a more distinctive indicator for replanning. As patient weights fluctuated considerably - due to side effects (weight loss) and saline volume expansion during chemotherapy (weight gain) - no significant correlations were found in our study.
For example, the plan recalculations for the patient with the most stable weight (overall loss: $2 \mathrm{~kg}$ ) did not lead to the most stable dose distributions for both techniques, judged by the evaluated dose criteria. Furthermore the underlying number of patients was not sufficient to derive correlations (e.g. with weight loss) with statistical significance.

Additionally, the anatomical changes cannot be viewed separately from daily set-up variations that are likely to have a large impact on the dose distributions as well. In our study, the main part of set-up variations is corrected by IGRT (resulting in couch shifts) and has therefore no impact on the dose distributions, i.e. their absolute values (applied couch shifts) are not relevant for our analysis. Nevertheless as not all variations can be corrected there are remaining set up-errors influencing the delivered doses. In comparison to the degree of impact caused by anatomical changes and weight loss these are considered to have a rather small effect though. Generally the impact of pure set-up uncertainties has been studied elsewhere, e.g. in the study by Kraan et al. [15].

When to adapt a plan, i.e. to define the conditions where a plan based on the current patient anatomy is no longer acceptable, will remain a question to be investigated in the future. Especially for IMPT the approach of this issue might be difficult, where impacts of

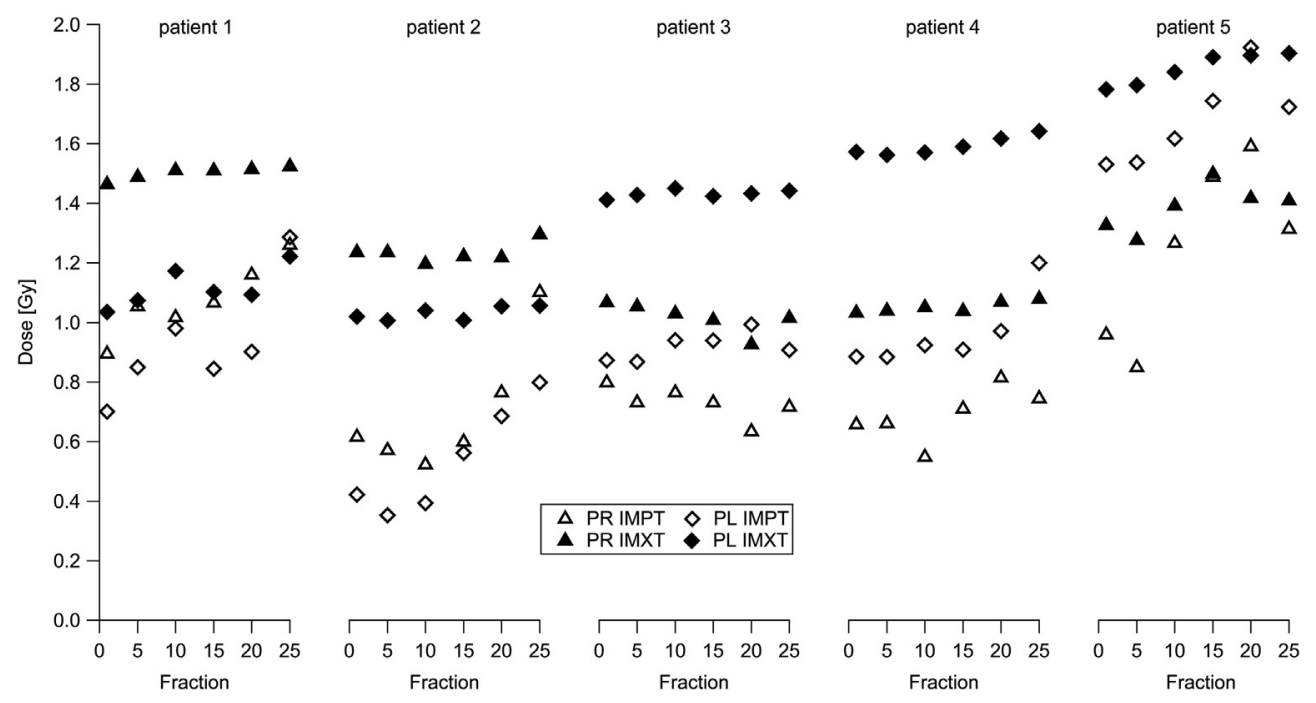

Figure 6. Trends of mean dose to left and right parotid gland (PL and PR) of five patients - each symbol represents one parotid and one modality. 


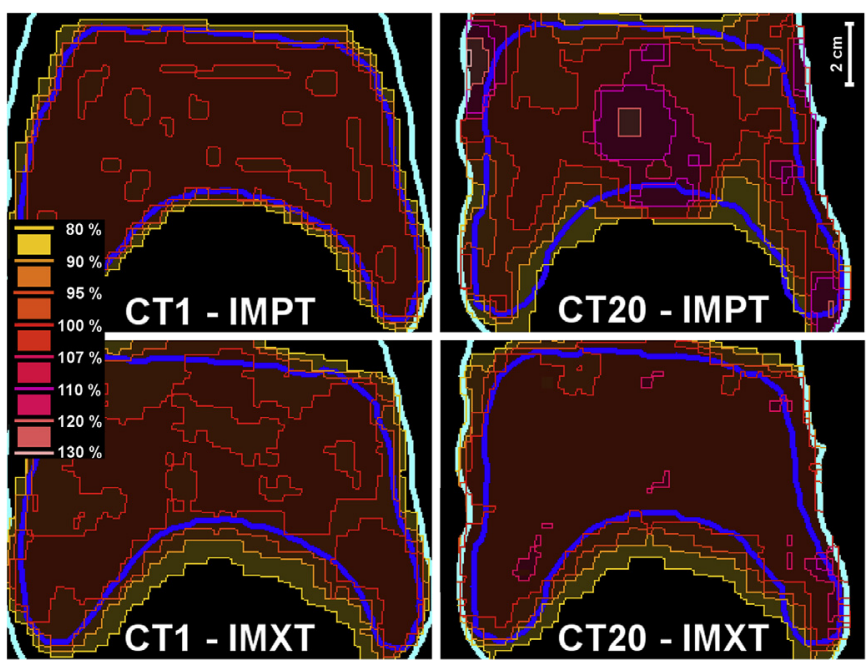

Figure 7. Dose distributions of the PTV area - initial plans (left) vs. recalculations (right) at 20th fraction for protons (top) and photons (bottom). (Normalization: $100 \%=2$ Gy; PTV contour: dark blue, patient contour: light blue).

misalignment are larger than in IMXT, as huge variations in dose between fractions implicated.

The resulting IMPT coverage values of as low as $66.7 \%$ lead to questions of clinical relevance and how this risk of underdosage should be dealt with. One has to note that this was a single fraction, and one has to take a closer look onto the calculated coverages over all fractions. Here we can only estimate worst cases, and mean coverages which were between $81 \%$ and $92 \%$ for IMPT. A more accurate statement would demand recalculations on daily control images and dose accumulation studies. Even though such extreme deviations like the above stated $66.7 \%$ were exceptions throughout the treatment period, they still show the need of accurate, high quality IGRT and frequent control CTs, especially in proton therapy.

The dosimetric evaluation was performed for the PTV (as motivated in the Methods section) of which rather the inner part (i.e. the CTV) is of clinical relevance. Regarding the location of cold spots, dose inhomogeneities of IMPT recalculations were spread all over the target volume, i.e. within the CTV as well, while for IMXT underdosage was more pronounced in the peripheral regions of the PTV, outside of the CTV. This indicates that PTV-based plan optimization does not account sufficiently for all uncertainties in IMPT which was also shown elsewhere [20].

Uncertainties in set-up and range are well-known issues for IMPT and can be reduced by so-called robust optimization [20,27-29]. Different robust optimization algorithms and methods can improve the sensitivity of IMPT plans in terms of set-up errors and range uncertainties by incorporating uncertainty information into the planning process. Conducting the study with robust optimization might lead to different dosimetric results. However, for our study we do not expect large differences in the dosimetric consequences and believe that the conclusion would be similar. The major impact on the dose distributions of our study is assumed to be caused by anatomical changes which are only partly taken into account in some of the algorithms by estimating the caused range uncertainties. For a detailed analysis, the study would have to be repeated on initial plans calculated using a robust optimization tool.

Explanations for the great differences in the impact of geometrical changes between IMPT and IMXT plans can be given by the different physical properties of the particles and corresponding features of plans. The great advantage of the protons, its finite range and high spatial precision, is at the same time a well-known issue
[14-20]. While changes of density and thickness of the traversed matter lead to a slight deviation in dose values for photons, the range of the protons is changed. Due to the applied intensity modulation and spot scanning technique each IMPT field consisted of spots of different weights and energies. Density changes can cause shifts of single spots and lead to unfavorable dose accumulations from neighboring spots.

Our study was done on daily high quality IGRT by the tomotherapy unit, correcting the patient set-up with a very high quality. Therefore our CT data represent almost the ideal case, with frequent high quality imaging. To put this into perspective for different clinical facilities of IMPT or IMXT it has to be emphasized that the quality of IGRT depends strongly on the imaging possibilities and their frequency, and that this might have a large impact on the dose distributions, which may vary from our results.

\section{Conclusion}

We investigated the impact of interfractional geometrical changes to dose distributions of IMPT and IMXT plans and demonstrated differences in the consequences of the two irradiation techniques. Although absolute alterations were larger in IMPT plans, doses to OARs were more critical for IMXT plans. Tumor coverage was less sensitive for IMXT plans; IMPT dose distributions indicated a high risk for local underdosage. The amount of dosimetric changes differed largely between the individual patients, in particular for IMPT plans.

For both modalities our data indicate the importance of frequent control imaging and recalculations of treatment plans on control CTs. Plan adaptions triggered by these recalculations might reduce the underdosage of the tumor (most relevant for IMPT) and the overdosage of OARs (in IMXT).

\section{Conflict of interest statement}

Severin Kampfer, Simeon Nill, Uwe Oelfke and Hans Geinitz have nothing to disclose.

Jan Wilkens and Birgit Müller report grants from German Research Foundation (DFG) during the conduct of the study. Jan Wilkens further reports grants from Brainlab AG, personal fees from ProHealth AG, outside the submitted work. Marciana Duma reports grants from Accuray, outside the submitted work.

\section{Acknowledgments}

Supported by DFG cluster of excellence: Munich-Centre for Advanced Photonics. We thank Rolf Bendl and the VIRTUOS developer team (German Cancer Research Center Heidelberg) for their support.

\section{References}

[1] Schwartz DL, Garden AS, Thomas J, Chen Y, Zhang Y, Lewin J, et al. Adaptive radiotherapy for head-and-neck cancer: initial clinical outcomes from a prospective trial. Int J Radiat Oncol Biol Phys 2012;83(3):986-93.

[2] Jensen AD, Nill S, Huber PE, Bendl R, Debus J, Münter MW. A clinical concept for interfractional adaptive radiation therapy in the treatment of head and neck cancer. Int J Radiat Oncol Biol Phys 2012;82(2):590-6.

[3] Simone II CB, Ly D, Dan TD, Ondos J, Ning H, Belard A, et al. Comparison of intensity-modulated radiotherapy, adaptive radiotherapy, proton radiotherapy, and adaptive proton radiotherapy for treatment of locally advanced head-and-neck cancer. Radiother Oncol 2011;101:376-82.

[4] Duma MN, Kampfer S, Wilkens JJ, Schuster T, Molls M, Geinitz H. Comparative analysis of an image-guided versus a non-image-guided setup approach in terms of delivered dose to the parotid glands in head-and-neck cancer IMRT. Int J Rad Oncol Biol Phys 2010;77(4):1266-73.

[5] Hansen EK, Bucci MK, Quivey JM, Weinberg V, Xia P. Repeat CT imaging and replanning during the course of IMRT for head-and-neck cancer. Int J Radiat Oncol Biol Phys 2006;64(2):355-62. 
[6] Barker Jr JL, Garden AS, Ang KK, O'Daniel JC, Wang H, Court LE, et al. Quantification of volumetric and geometric changes occurring during fractionated radiotherapy for head-and-neck cancer using an integrated CT/linear accelerator system. Int J Radiat Oncol Biol Phys 2004;59(4):960-70.

[7] Ho KF, Marchant T, Moore C, Webster G, Rowbottom C, Penington H, et al. Monitoring dosimetric impact of weight loss with kilovoltage $(\mathrm{kV})$ cone beam CT (CBCT) during parotid-sparing IMRT and concurrent chemotherapy. Int J Radiat Oncol Biol Phys 2012;82(3):e375-82.

8] Ramaekers BLT, Grutters IPC, Pijls-Johannesma M, Lambin P, Joore MA Langendijk JA. Protons in head and neck cancer: bridging the gap of evidence. Int J Radiat Oncol Biol Phys 2013;85(5):1282-8.

[9] Mendenhall NP, Malyapa RS, Zhong S, Yeung D, Mendenhall WM, Li Z. Proton therapy for head and neck cancer: rationale, potential indications, practical considerations, and current clinical evidence. Acta Oncol 2011;50:763-71.

[10] Chan AW, Liebsch NJ. Proton radiation therapy for head and neck cancer. J Surg Oncol 2008;97:697-700.

[11] Taheri-Kadkhoda Z, Bijörk-Eriksson T, Nill S, Wilkens JJ, Oelfke U, Johansson K, et al. Intensity-modulated radiotherapy of nasopharyngeal carcinoma: a comparative treatment planning study of photons and protons. Radiat Oncol 2008;3:4.

[12] Steneker M, Lomax AJ, Schneider U. Intensity modulated photon and proton therapy for the treatment of head and neck tumors. Radiother Oncol 2006;80: 263-7.

[13] Cozzi L, Fogliata A, Lomax A, Bolsi A. A treatment planning comparison of 3D conformal therapy, intensity modulated photon therapy and proton therapy for treatment of advanced head and neck tumours. Radiother Oncol 2001;61: 287-97.

[14] Lomax T. Towards daily adapted proton therapy. Phys Med 2014;30(Suppl. 1 ):e3.

[15] Kraan AC, van de Water S, Teguh DN, Al-Mamgani A, Madden T, Kooy HM, et al. Dose uncertainties in IMPT for oropharyngeal cancer in the presence of anatomical, range, and setup errors. Int J Radiat Oncol Biol Phys 2013;87(5): 888-96.

[16] McGowan SE, Burnet NG, Lomax AJ. Treatment planning optimisation in proton therapy. Brit J Radiol 2013:86. 20120288.

[17] Lassen-Ramshad Y, Vestergaard A, Muren LP, Hoyer M, Petersen JBB. Plan robustness in proton beam therapy of a childhood brain tumour. Acta Oncol 2011:50:791-6.
[18] Lomax AJ. Intensity modulated proton therapy and its sensitivity to treatment uncertainties 2: the potential effects of inter-fraction and inter-field motions. Phys Med Biol 2008;53:1043-56.

[19] Albertini F, Bolsi A, Lomax AJ, Rutz HP, Timmerman B, Goitein G. Sensitivity of intensity modulated proton therapy plans to changes in patient weight. Radiother Oncol 2008;86:187-94.

[20] Unkelbach J, Bortfeld T, Martin BC, Soukup M. Reducing the sensitivity of IMPT treatment plans to setup errors and range uncertainties via probabilistic treatment planning. Med Phys 2009;36(1):149-63.

[21] Grégoire V, Eisbruch A, Hamoir M, Levendag P. Proposal for the delineation of the nodal CTV in the node-positive and the post-operative neck. Radiother Oncol 2006;79:15-20.

[22] Chao KSC, Wippold II FJ, Ozyigit G, Tran BN, Dempsey JF. Determination and delineation of nodal target volumes for head-and-neck cancer based on patterns of failure in patients receiving definitive and postoperative IMRT. Int Radiat Oncol Biol Phys 2002;53(5):1174-84.

[23] Nill S, Bortfeld T, Oelfke U. Inverse planning of intensity modulated proton therapy. Z Med Phys 2004;14:35-40.

[24] Newhauser WD, Giebeler A, Langen KM, Mirkovic D, Mohan R. Can megavoltage computed tomography reduce proton range uncertainties in treatment plans for patients with large metal implants? Phys Med Biol 2008;53: $2327-44$.

[25] Lomax AJ. Intensity modulation methods for proton radiotherapy. Phys Med Biol 1999;44:185-205.

[26] Grzadziel A, Grosu A, Kneschaurek P. Three-dimensional conformal versus intensity-modulated radiotherapy dose planning in stereotactic radiotherapy: application of standard quality parameters for plan evaluation. Int J Radiat Oncol Biol Phys 2006;66(4):S87-94 [Suppl.].

[27] Liu W, Frank SJ, Xiaoqiang L, Yupeng L, Zhu RX, Mohan R. PTV-based IMPT optimization incorporating planning risk volumes vs robust optimization. Med Phys 2013;40:021709. http://dx.doi.org/10.1118/1.4774363.

[28] Pflugfelder D, Wilkens JJ, Oelfke U. Worst case optimization: a method to account for uncertainties in the optimization of intensity modulated proton therapy. Phys Med Biol 2008;53:1689-700.

[29] Chen W, Unkelbach J, Trofimov A, Madden T, Kooy H, Bortfeld T, et al Including robustness in multi-criteria optimization for intensity-modulated proton therapy. Phys Med Biol 2012;57:591-608. 